\title{
ESTUDO DA ASSOCIAÇÃO ENTRE ÓXIDOS DE NITROGÊNIO E CONCENTRAÇÃO DE OZÔNIO COM PARÂMETROS METEOROLÓGICOS
}

\author{
Amaury De Souza ${ }^{1}$ \\ Flavio Aristone ${ }^{2}$ \\ Ana Paula Garcia ${ }^{3}$ \\ Debora A S Santos ${ }^{4}$
}

\begin{abstract}
Resumo: O presente estudo investiga as relações das medidas tanto da concentração de ozônio $\left(\mathrm{O}_{3}\right)$ como de óxidos de nitrogênio $\left(\mathrm{NO}_{\mathrm{x}}=\mathrm{NO}+\mathrm{NO}_{2}\right)$ em função das condições meteorológicas para a atmosfera de Campo Grande, MS, no período compreendido de janeiro a dezembro de 2015. Variações diurnas e sazonais de poluentes gasosos foram investigadas e comparadas usando os resultados da análise de séries temporais. Os ciclos diurnos de $\mathrm{O}_{3}$ e de $\mathrm{NO}_{x}$ se relacionam inversamente com o $\mathrm{O}_{3}$ superficial, que apresenta picos ao meio dia, e menores concentrações noturnas. Os resultados mostram que as concentrações máximas de $\mathrm{O}_{3}$ e oxidantes $\left(\mathrm{O}_{\mathrm{x}}=\mathrm{O}_{3}+\mathrm{NO}_{\mathrm{x}}\right)$ em Campo Grande geralmente aparecem no início da tarde, por volta das 15:00 horas. A variação diária das concentrações de NO mostra um ciclo muito claro com dois picos, um aparecendo por volta das 07:00 e outro às 11:00 horas. No nível mais baixo, o $\mathrm{NO}_{2}$ é o principal componente do $\mathrm{NO}_{x}$, enquanto o $\mathrm{NO}$ domina a maior taxa de mistura. Fortes correlações positivas foram observadas entre o $\mathrm{O}_{3}$ com a temperatura e a radiação solar, e com forte correlação negativa com a umidade relativa do ar, durante o período estudado.
\end{abstract}

Palavras-chave: Óxidos de nitrogênio; Ozônio; Sazonalidade; Tendência

\section{STUDY OF THE ASSOCIATION BETWEEN NITROGEN OXIDES AND OZONE CONCENTRATION WITH METEOROLOGICAL PARAMETERS}

\begin{abstract}
The present study investigates the relationship between both the ozone concentration $\left(\mathrm{O}_{3}\right)$ and nitrogen oxides $\left(\mathrm{NO}_{\mathrm{x}}=\mathrm{NO}+\mathrm{NO}_{2}\right)$ as a function of meteorological conditions for the atmosphere of Campo Grande, MS, from January to December, 2015. Daily as well as seasonal effects of pollutant gases were investigated and compared using results of time series analysis. The $\mathrm{O}_{3}$ and $\mathrm{NO}_{x}$ diurnal cycles are inversely related to the superficial $\mathrm{O}_{3}$, which presents peaks at noon and lower nocturnal concentrations. The results show that the maximum concentrations of $\mathrm{O}_{3}$ and oxidants $\left(\mathrm{O}_{\mathrm{x}}=\mathrm{O}_{3}+\mathrm{NO}_{\mathrm{x}}\right)$ in Campo Grande usually appear in the early afternoon, around 3:00 P. M. The daily variation of NO concentration shows a very clear cycle with two peaks, the first one appearing around 07:00 a.m. and the second at 11:00 a.m. At the lowest level of $\mathrm{NO}_{\mathrm{x}}, \mathrm{NO}_{2}$ is the main component, while $\mathrm{NO}$ dominates the highest blend rate. Strong positive correlations were observed between $\mathrm{O}_{3}$ and

\footnotetext{
${ }^{1}$ Dr. em Tecnologia Ambientais, Universidade Federal de Mato Grosso do Sul. Email: amaury.de@uol.com.br

${ }^{2}$ Dr. em Génie Physique pelo Institut National des Sciences Appliquées de Toulouse, Universidade Federal de Mato Grosso do Sul. Email: flavio.aristone@ufms.br

${ }^{3}$ Dra. em Tecnologia Ambientais, Universidade De Guarulhos. Email: apg.bio@gmail.com

${ }^{4}$ Dra em Recursos Naturais, Universidade Federal de Mato Grosso, campus de Rondonopolis. Email: deboraassantos@hotmail.com
} 
temperature and between $\mathrm{O}_{3}$ and solar radiation, as well as a strong negative correlation was observed between $\mathrm{O}_{3}$ and the relative humidity of the air, during the studied period.

Keywords: Nitrogen oxides; Ozone; Seasonality; Trend

\section{ESTUDIO DE LA ASOCIACIÓN ENTRE ÓXIDOS DE NITROGENO Y CONCENTRACIÓN DE OZONO CON PARÁMETROS METEOROLÓGICOS}

Resumen: El presente estudio investiga la relación entre tanto la concentración de ozono (O3) y el nitrógeno $(\mathrm{NOx}=\mathrm{NO}+\mathrm{NO} 2)$ a la función de condiciones meteorológicas para el ambiente de Campo Grande, MS, de enero a diciembre de 2015. Daily así como los efectos adversos de los agentes contaminantes fueron investigados y comparados con resultados de análisis de tiempo. Los $\mathrm{O} 3$ y los NOx diurnal ritmos son inversamente relacionados con la superficie superficial O3, que presenta peaks at noon and lower nocturnal concentraciones. Los resultados muestran que las concentraciones máximas de $\mathrm{O} 3$ y oxidantes $(\mathrm{Ox}=\mathrm{O} 3+$ NOx) en Campo Grande suelen aparecer en la temprana temprana, alrededor de las 3:00 PM La secuencia diaria de NO de la concentración muestra un cambio de ciclo con dos puntos, el primero En el nivel inferior de $\mathrm{NOx}, \mathrm{NO} 2$ es el componente principal, mientras que no dominan la tasa de mezcla más alta. Las correlaciones positivas positivas se observaron entre O3 y la temperatura y entre O3 y las radares solares, así como una fuerte correlación se observó entre $\mathrm{O} 3$ y la relativa relativa del aire durante el período de consulta.

Palabras clave: Oxido de nitrógeno; Ozono; Estacionalidad; Tendencia

\section{INTRODUÇÃO}

O ozônio $\left(\mathrm{O}_{3}\right)$ e os óxidos de nitrogênio $\left(\mathrm{NO}_{\mathrm{x}}\right)$, compreendendo dióxido de nitrogênio $\left(\mathrm{NO}_{2}\right)$ e óxido nítrico $(\mathrm{NO})$, estão entre os contaminantes mais importantes nas áreas urbanas, pois foram associados a efeitos adversos na saúde humana e no ambiente natural (Hagenbjörk et $a l$, 2017). Os veículos automotores são as principais fontes de $\mathrm{NO}_{\mathrm{x}}$ nos grandes centros urbanos. A principal proporção de $\mathrm{NO}_{\mathrm{x}}$ é emitida como $\mathrm{NO}$, enquanto uma proporção menor é emitida diretamente como $\mathrm{NO}_{2}$. Mesmo que a quantidade total de $\mathrm{NO}_{\mathrm{x}}$ tenha uma tendência descendente, a participação de $\mathrm{NO}_{2}$ nas emissões de $\mathrm{NO}_{\mathrm{x}}$ aumentou nos últimos anos, pois depende do veículo, tipo de combustível utilizado, tecnologia de tratamento de exaustão e condições de condução (Carslaw et al, 2005). Na cidade de Campo Grande, localizada no estado de Mato Grosso do Sul, a concentração de ozônio medida ao nível do solo foi iniciada em 2004. A concentração de ozônio tem mostrado uma tendência crescente no estado de Mato Grosso do Sul, desde as primeiras medições em 2004, e este tem sido o principal poluente em muitas áreas do estado (Souza et al, 2018; Souza e Fernandes, 2014; Pires et al, 2014).

Uma vez que a maioria das políticas existentes para reduzir os níveis troposféricos de $\mathrm{O}_{3}$ nas áreas urbanas se concentra na redução das emissões de precursores, o seu sucesso depende fortemente no desenrolar, com precisão, da sensibilidade da produção de $\mathrm{O}_{3}$. A 
União Europeia (UE) estabeleceu normas de qualidade do ar em relação à concentração de ozônio ambiente. A diretiva 2008/50 define informação e limiares de alerta que se referem a valores horários igual a 180 e $240 \mathrm{mg} / \mathrm{m}^{3}$. A mesma diretiva também define uma orientação para a proteção da saúde humana de forma clara: o valor médio máximo obtido para 8 horas diárias não deve exceder o valor alvo de $120 \mathrm{mg} / \mathrm{m}^{3}$ por mais de 25 dias durante o ano civil, em um período de três anos (EC 2008). No Brasil, a Resolução do Conama de 28 de julho de 1990 define poluição atmosférica e estabelece como limite de poluição "qualquer forma de matéria ou energia com intensidade e em quantidade, concentração, tempo ou características em desacordo com os níveis estabelecidos, que tornem ou possam tornar o ar impróprio, nocivo ou ofensivo à saúde, inconveniente ao bem-estar público, capaz de causar danos aos materiais, à fauna e a flora, que seja prejudicial à segurança, ao uso e ao gozo da propriedade e às atividades normais da comunidade" (Brasil, MMA/Conama, 1990).

O presente estudo tem como objetivo investigar as relações das medidas de concentração do ozônio $\left(\mathrm{O}_{3}\right)$ e óxidos de nitrogênio $\left(\mathrm{NO}_{\mathrm{x}}=\mathrm{NO}+\mathrm{NO}_{2}\right)$, juntamente com as condições meteorológicas para a atmosfera de Campo Grande, MS, no período de janeiro a dezembro de 2015.

\section{MÉTODOS}

\section{Área de estudo e dados observacionais}

Campo Grande é a capital do estado de Mato Grosso do Sul (MS) e está situada no centro do Estado, na parte sul da região Centro-Oeste do Brasil. Geograficamente, o município de Campo Grande se situa próximo das fronteiras do Brasil com Paraguai e Bolívia. Localiza-se na latitude de 20²6’34” Sul e longitude de 54³8’47” Oeste. Ocupa uma superfície total de $8.096,051 \mathrm{~km}^{2}$, que corresponde a 2,26\% da área total do Estado. A área urbana totaliza $154,45 \mathrm{~km}^{2}$.

Os principais problemas de poluição na cidade são atribuídos ao tráfego de veículos, à taxa de aumento da atividade de construção, aos depósitos de lixo, ao uso de pequenos geradores de energia usados para suprir a falta de energia eléctrica, e às queimadas intencionais praticadas para limpeza de terrenos (Souza et al, 2010). A figura 1 representa a localização desse Município e a estação de monitoramento contínuo de ar localizada no campus da Universidade Federal do Sul do Mato Grosso (UFMS). 


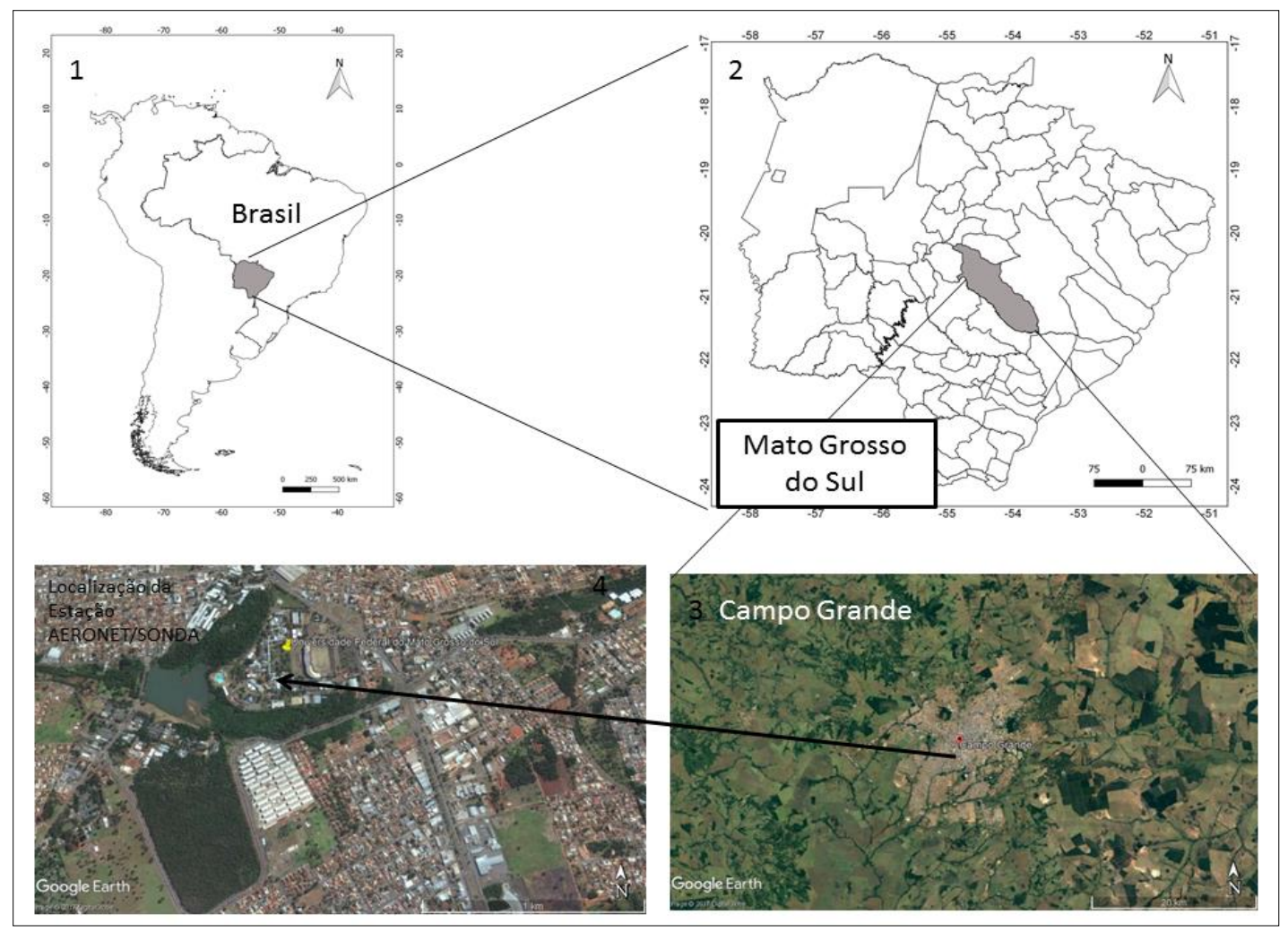

Figura 1. Localização do município de Campo Grande no estado do Mato Grosso do Sul, e a estação de monitoramento contínuo de ar localizada no campus da Universidade Federal do Sul do Mato Grosso.

\section{Coleta de Dados}

A Tabela 1 mostra as medias dos valores obtidos médios para os parâmetros meteorológicos considerados.

Tabela 1. Dados meteorológicos para o período de amostragem (2015).

\begin{tabular}{|c|c|c|c|c|c|c|}
\hline Variável & Condição & Unidade & Verão & Outono & Inverno & Primavera \\
\hline \multirow{3}{*}{ Temperatura } & mínima & \multirow{3}{*}{${ }^{\circ} \mathrm{C}$} & 21,59 & 14,7 & 12,74 & 15,68 \\
\hline & media & & 25,22 & 22,51 & 22,82 & 25,61 \\
\hline & máxima & & 28,25 & 26,1 & 42 & 30,36 \\
\hline
\end{tabular}




\begin{tabular}{|c|c|c|c|c|c|c|}
\hline \multirow{3}{*}{ Umidade } & mínima & \multirow{3}{*}{$\%$} & 29,8 & 30,8 & 14,9 & 32,8 \\
\hline & media & & 77,95 & 82,81 & 79,52 & 88,24 \\
\hline & máxima & & 98,4 & 98,5 & 98,5 & 98,4 \\
\hline \multirow{3}{*}{$\begin{array}{l}\text { Pressão } \\
\text { atmosférica }\end{array}$} & mínima & \multirow{3}{*}{ mbar } & 907 & 905,7 & 904,3 & 903,3 \\
\hline & media & & 912,99 & 915,49 & 914,57 & 912,48 \\
\hline & máxima & & 918,6 & 925,8 & 919,9 & 919,2 \\
\hline \multirow{3}{*}{$\begin{array}{l}\text { Velocidade dos } \\
\text { ventos }\end{array}$} & mínima & \multirow{3}{*}{$\mathrm{m} / \mathrm{s}$} & 0,2 & 0,1 & 0,1 & 0,1 \\
\hline & media & & 1,93 & 1,77 & 1,75 & 2,16 \\
\hline & máxima & & 6,4 & 7,6 & 7,5 & 6,7 \\
\hline \multirow{3}{*}{ Direção dos ventos } & mínima & \multirow{3}{*}{ graus } & 10,3 & 4,3 & 6,6 & 7,9 \\
\hline & media & & 158,18 & 149,06 & 138,46 & 140,37 \\
\hline & máxima & & 347,9 & 354 & 354 & 350,6 \\
\hline \multirow{3}{*}{ Radiação solar } & mínima & \multirow{3}{*}{$\mathrm{W} / \mathrm{m}^{2}$} & 0 & 0 & 0 & 0 \\
\hline & media & & 169,62 & 96,58 & 125,3 & 116,68 \\
\hline & máxima & & 973,5 & 839,5 & 793,6 & 935,9 \\
\hline \multirow{3}{*}{$\begin{array}{l}\text { Radiação } \\
\text { Ultravioleta }\end{array}$} & mínima & \multirow{3}{*}{$\mathrm{W} / \mathrm{m}^{2}$} & 0 & 0 & 0,01 & 0 \\
\hline & média & & 7,6 & 3,83 & 4,14 & 5,3 \\
\hline & máxima & & 40,26 & 28,85 & 28,27 & 34,23 \\
\hline
\end{tabular}

Fonte: CEMTEC-MS

\section{Análise estatística dos dados}

Foram calculadas as concentrações horárias médias dos valores de $\mathrm{NO}, \mathrm{NO}_{\mathrm{x}}, \mathrm{NO}_{2}$ e $\mathrm{O}_{3}$ para cada uma das quatro estações do ano. Além disso, as variações diárias médias dos poluentes estudados foram calculadas. Foram realizadas análises de correlação entre as concentrações médias horárias de $\mathrm{O}_{3}, \mathrm{NO}, \mathrm{NO}_{\mathrm{x}}$ e $\mathrm{O}_{\mathrm{x}}$ com os parâmetros meteorológicos. Um gráfico de dispersão da variação da média horária diária $\left[\mathrm{NO}_{2}\right] /\left[\mathrm{O}_{\mathrm{x}}\right]$ em função da 
concentração de $\mathrm{NO}_{\mathrm{x}}$ foi utilizado para fornecer uma melhor visão geral e interpretação das relações entre o $\mathrm{NO}_{x}$ e os poluentes $\mathrm{NO}_{2}, \mathrm{NO}_{\mathrm{x}}$ e $\mathrm{O}_{3}$. A Tabela 2 mostra a instrumentação usada para medir poluentes atmosféricos e parâmetros meteorológicos.

Tabela 2. Instrumentação usada para medir poluentes atmosféricos e parâmetros meteorológicos durante o ano de 2015 em Campo Grande.

\begin{tabular}{|c|c|c|c|c|}
\hline Parâmetro & $\begin{array}{l}\text { Modelo do } \\
\text { instrumento }\end{array}$ & Detector & $\begin{array}{l}\text { Número de } \\
\text { Método } \\
\text { Equivalente da } \\
\text { PA }\end{array}$ & Erro \\
\hline $\mathrm{O}_{3}$ & $\begin{array}{l}\text { ThermoEnvironmental } \\
49 \mathrm{C}\end{array}$ & $\begin{array}{l}\text { Chemiluminesce } \\
\text { nce }\end{array}$ & $\begin{array}{l}\text { EQOA-0880- } \\
047\end{array}$ & $1 \mathrm{ppb}$ \\
\hline $\begin{array}{l}\mathrm{NO}, \mathrm{NO}_{2} \\
\text { e } \mathrm{NO}_{\mathrm{X}}\end{array}$ & $\begin{array}{l}\text { ThermoEnvironmental } \\
42 \mathrm{C}\end{array}$ & $\begin{array}{l}\text { Chemiluminesce } \\
\text { nce }\end{array}$ & $\begin{array}{l}\text { RFNA-1289- } \\
074\end{array}$ & $\begin{array}{l}0,4 \\
\mathrm{ppb}\end{array}$ \\
\hline $\mathrm{SO}_{2}$ & $\begin{array}{l}\text { ThermoEnvironmental } \\
43 \mathrm{C}\end{array}$ & Fluorescence & $\begin{array}{l}\text { EQSA-0486- } \\
060\end{array}$ & $1 \mathrm{ppb}$ \\
\hline $\begin{array}{l}\text { Velocidad } \\
\text { e dos } \\
\text { ventos }\end{array}$ & Met One 010C & Anemometer & N.A. & $1 \%$ \\
\hline $\begin{array}{l}\text { Direção } \\
\text { dos ventos }\end{array}$ & Met One 020C & Potentiometer & N.A. & $3^{\circ}$ \\
\hline $\begin{array}{l}\text { Temperat } \\
\text { ura }\end{array}$ & Met One 060A & $\begin{array}{l}\text { Multistage } \\
\text { thermistor }\end{array}$ & N.A. & $0,5^{\circ} \mathrm{C}$ \\
\hline Pressão & Met One 090D & $\begin{array}{l}\text { Barometric } \\
\text { sensor }\end{array}$ & N.A. & $\begin{array}{l}1,35 \\
\text { mbar }\end{array}$ \\
\hline RH & Met One 083E & $\begin{array}{l}\text { Capacitance } \\
\text { sensor }\end{array}$ & N.A. & $2 \%$ \\
\hline SR & Met One 095 & Pyranometer & N.A. & $1 \%$ \\
\hline
\end{tabular}




\section{RESULTADOS E DISCUSSÃO}

A velocidade dos ventos foi maior na primavera e menor no verão, outono e inverno, com a taxa média ligeiramente inferior às normais climatológicas. A velocidade média foi de $1,90 \mathrm{~m} / \mathrm{s}$ com um mínimo de 0,10 e máximo de 7,90 m/s. A pressão atmosférica foi maior no outono e no inverno, com valores ligeiramente abaixo das normais climatológicas.

As temperaturas médias (Tabela 1) apresentaram comportamento semelhante às normais climatológicas. As temperaturas (média, máxima e mínima) no verão foram cerca de 9 a $10^{\circ} \mathrm{C}$ maiores do que as do inverno. A temperatura diária máxima média no verão foi de $26^{\circ} \mathrm{C}$ e no inverno de $21^{\circ} \mathrm{C}$, enquanto a temperatura média mínima diária no verão foi de $21^{\circ}$ $\mathrm{C}$ e $12^{\circ} \mathrm{C}$ no inverno. Esse mesmo intervalo entre as temperaturas máxima e máxima diária foi observado em todas as estações.

A umidade relativa estava ligeiramente abaixo das normais climatológicas, e não mostrou muita variação entre as diferentes estações. No entanto, a variação entre as médias diárias de umidade relativa máxima e mínima foi de $46 \%$ no verão e $38 \%$ no inverno.

\section{Variabilidade da série temporal de $\mathrm{NO}_{x}$ e $\mathrm{O}_{3}$}

A variabilidade das concentrações de poluentes atmosféricos depende de emissões específicas e condições meteorológicas gerais. $\mathrm{NO}_{\mathrm{x}}\left(\mathrm{NO}_{2}+\mathrm{NO}\right)$ é um contaminante primário e $\mathrm{O}_{3}$ é um contaminante secundário que se origina na atmosfera através de um conjunto de reações complexas (Seinfeld e Pandis, 2006). As concentrações de $\mathrm{O}_{3}$ são influenciadas por precursores de $\mathrm{NO}_{\mathrm{x}}$ e condições meteorológicas (temperatura e radiação solar). No presente trabalho foram examinadas séries temporais de concentrações de poluentes atmosféricos (NO, $\mathrm{NO}_{2}, \mathrm{NO}_{\mathrm{x}}$ e $\mathrm{O}_{3}$ ) para o período de estudo de um ano (1 ano). As tendências das séries indicam as seguintes variações: $\mathrm{SO}_{2}$ estável; $\mathrm{NO}$ estável; $\mathrm{NO}_{2}$ crescente; $\mathrm{NO}_{\mathrm{x}}$ levemente crescente; $\mathrm{O}_{\mathrm{x}}$ levemente crescente; $\mathrm{O}_{3}$ crescente.

As concentrações elevadas de $\mathrm{NO}_{\mathrm{x}}$ e $\mathrm{NO}$ foram observadas durante todo o período estudado. Além disso, os resultados apresentam maiores concentrações de NO, o que pode ser atribuído à maior influência veicular, caracterizada por frota "pesada". Na Figura 2 se pode ver os níveis aumentados de concentração de $\mathrm{O}_{3}$, provavelmente devido à diminuição dos níveis de $\mathrm{NO}$ e, consequentemente, ao menor consumo de $\mathrm{O}_{3}$, produzindo um acúmulo de $\mathrm{O}_{3}$ troposférico. $\mathrm{O}$ aumento dos níveis de $\mathrm{NO}$ e $\mathrm{NO}_{x}$ podem causar uma redução de $\mathrm{O}_{3}$. Em uma atmosfera limitada de $\mathrm{NO}_{\mathrm{x}}$ (com altas concentrações de $\mathrm{NO}_{\mathrm{x}}$ ), o $\mathrm{O}_{3}$ pode depender do nível 
global de $\mathrm{NO}_{\mathrm{x}}$ (Seinfeld e Pandis, 2006). Além disso, a concentração ambiente de $\mathrm{O}_{3}$ é fortemente influenciada pela flutuação diária do $\mathrm{NO}_{2}$ e a proporção de $\mathrm{NO}_{2}$ para $\mathrm{NO}$ (Sebald et al, 2000).

A variação sazonal desses poluentes pode ser melhor avaliada através dos resultados apresentados na Tabela 3, onde estão descritas as concentrações médias de $\mathrm{NO}, \mathrm{NO}_{\mathrm{x}}, \mathrm{NO}_{2}$ e $\mathrm{O}_{3}$, no inverno, primavera, verão e outono. Em geral, os níveis de concentrações de óxidos de nitrogênio ( $\mathrm{NO}, \mathrm{NO}_{2}, \mathrm{NO}_{\mathrm{x}}$ ) nas estações frias (outono e inverno) e de $\mathrm{O}_{3}$ nas estações quentes (verão e primavera) foram maiores. As maiores concentrações de $\mathrm{O}_{3}$ durante a primavera e o verão foram consequências da temperatura favorável e abundância de radiação solar, promovendo as reações fotoquímicas.

As concentrações mais baixas de $\mathrm{O}_{3}$ durante os meses mais frios podem ser atribuídas à presença de maiores concentrações de $\mathrm{NO}, \mathrm{NO}_{2}$ e $\mathrm{NO}_{\mathrm{x}}$ e condições climáticas específicas, como radiação solar mais baixa, menor camada de mistura e ventos fracos (Notario et al, 2012). Nesses dias mais frios, a formação fotoquímica de $\mathrm{O}_{3}$ é inibida pela falta de radiação solar intensa (Sadanaga et al, 2008; Geddes et al, 2009).

Tabela 3 -Valores médios, máximos e mínimos para as estacoes do ano para os parâmetros de poluição estudados em 2015.

\begin{tabular}{cccccccc}
\hline $\begin{array}{c}\text { Estacoes/variáveis } \\
(\mathrm{ppb})\end{array}$ & & $\mathrm{SO}_{2}$ & $\mathrm{NO}$ & $\mathrm{NO}_{2}$ & $\mathrm{NOx}$ & $\mathrm{OX}$ & $\mathrm{O}_{3}$ \\
& & & & & & & \\
verão & MAX & 33,61 & 3,36 & 4,64 & 8,00 & 22,96 & 18,32 \\
& MIN & 0,00 & 0,10 & 0,10 & 0,90 & 5,30 & 1,90 \\
& & & & & & & \\
\multirow{2}{*}{ outono } & MEDIA & 0,94 & 10,12 & 2,84 & 12,96 & 16,56 & 13,72 \\
& MAX & 18,30 & 165,00 & 28,10 & 165,00 & 63,50 & 61,20 \\
& MIN & 0,00 & 0,00 & 0,00 & 0,00 & 2,10 & 2,00 \\
& & & & & & & \\
& MEDIA & 1,96 & 8,41 & 7,45 & 15,86 & 23,50 & 16,05 \\
& MAX & 46,60 & 144,80 & 60,20 & 144,80 & 71,30 & 57,70
\end{tabular}




\begin{tabular}{cccccccc} 
& MEDIA & 2,24 & 6,25 & 7,64 & 13,89 & 23,97 & 16,33 \\
primavera & MAX & 14,70 & 57,30 & 51,70 & 100,60 & 65,70 & 47,70 \\
& MIN & 0,10 & 0,00 & 0,01 & 1,46 & 5,42 & 1,00 \\
\hline
\end{tabular}

Fonte: UFMS

Na Figura 2 estão representados os resultados obtidos para as medidas da variação diária das concentrações médias horárias de $\mathrm{NO}, \mathrm{NO}_{2}, \mathrm{NO}_{x}, \mathrm{O}_{\mathrm{x}}$ e $\mathrm{O}_{3} . \mathrm{NO}_{2}$ e $\mathrm{O}_{3}$ podem ser observados em conjunto como $\mathrm{O}_{\mathrm{x}}\left(\mathrm{O}_{\mathrm{x}}=\mathrm{NO}_{2}+\mathrm{O}_{3}\right)$, isto é, a quantidade total de oxidantes fotoquímicos. $\mathrm{Na}$ verdade, o $\mathrm{O}_{\mathrm{x}}$ é uma representação mais correta, pois é menos sensível às emissões e suas incertezas. $\mathrm{O}_{\mathrm{x}}$ não é influenciado pelo equilíbrio foto-estacionário rápido entre $\mathrm{NO}, \mathrm{NO}_{2} \mathrm{e}_{3}$ (Monteiro et al, 2005). A concentração de $\mathrm{O}_{\mathrm{x}}$ no período estudado aumenta lentamente após o nascer do Sol (Figura 2), atinge um máximo durante o dia e diminui até a manhã seguinte. $\mathrm{O}$ aumento da concentração de $\mathrm{O}_{\mathrm{x}}$ é devido à formação de $\mathrm{O}_{3}$ fotoquímica.

Han et al (2011) relataram que a variação de $\mathrm{O}_{\mathrm{x}}$ durante o dia e a noite pode ser esperada se os processos fotoquímicos influenciarem os níveis de $\mathrm{O}_{\mathrm{x}}$ em áreas poluídas. A variação na superfície de $\mathrm{O}_{3}$, dentro de um dia, pode ser útil na delineação dos processos responsáveis pela formação de $\mathrm{O}_{3}$ em um local particular (Singla et al, 2011). 


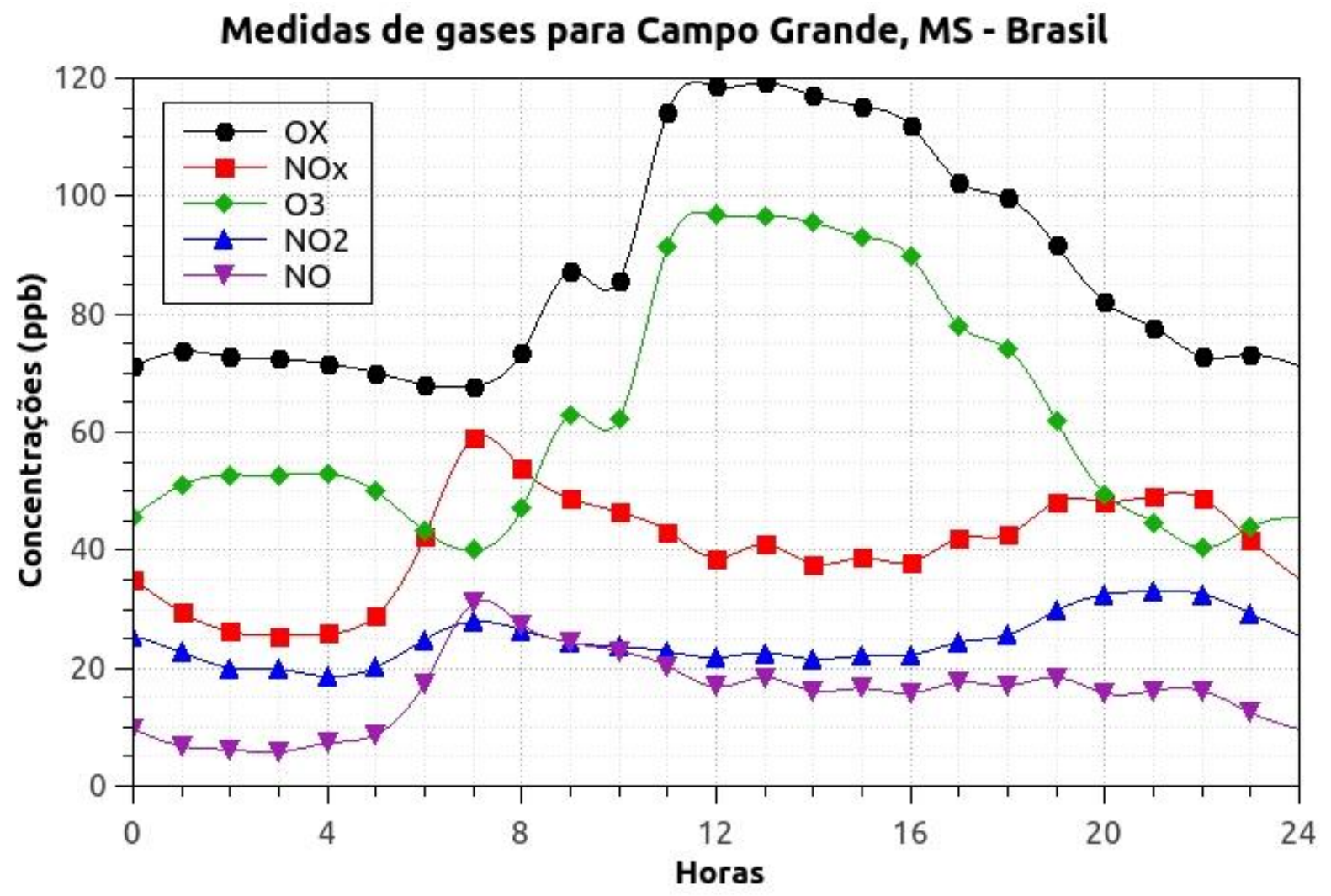

Figura 2. Variação diária horaria dos valores médios da concentração de $\mathrm{SO}_{2}, \mathrm{NO}, \mathrm{NO}_{2}, \mathrm{NO}_{\mathrm{x}}$ e $\mathrm{O}_{3}$ (tempo de média: 1 hora).

O NO, gás emitido majoritariamente pela exaustão de veículos, se mostra presente em concentrações elevadas, conforme os dados exibidos na Figura 2, tendo apresentado valores de pico igual a 10,97 ppb às 08:00. As concentrações máximas de $\mathrm{NO}_{2}$ foram de 8,85 também às 8:00. No período da manhã, o $\mathrm{NO}_{2}$ é produzido por oxidação de $\mathrm{NO}$ (Jenkin e Clemitshaw, 2000), pois o $\mathrm{NO}$ pode ser convertido em $\mathrm{NO}_{2}$ na presença de radicais de peróxidos (Dallarosa et al, 2007). Durante os períodos noturnos, as concentrações de $\mathrm{NO}$ e $\mathrm{NO}_{2}$ apresentaram um ligeiro aumento causado pelo aumento do tráfego veicular no horário de ponta, ou seja, às 18:00. Na sequência se dá a estabilidade da camada limite noturna. Nesse momento, o $\mathrm{NO}_{2}$ atingiu picos às 19:00 e às 20:00, da ordem de 10 ppB. Essas concentrações foram semelhantes aos picos exibidos pela manhã. Jenkin e Clemitshaw (2000) relataram que, à noite, o radical $\mathrm{OH}$ pode ser ignorado, pois é produzido principalmente a partir da fotólise de moléculas estáveis. Portanto, o $\mathrm{NO}_{2}$ não pode ser fotolisado para regenerar $\mathrm{NO}$, ou removido por reação com $\mathrm{OH}$, que reagirá com $\mathrm{O}_{3}$ para formar $\mathrm{NO}_{3}$, removendo assim $\mathrm{O}_{3}$. 
Da mesma forma, a Figura 2 exibe um aumento nas concentrações de $\mathrm{O}_{3}$ durante o dia, começando às 8:00 e atingindo seu máximo às 14:00, quando atinge o valor de 26,99 ppB. $\mathrm{O}$ $\mathrm{NO}$ é convertido em $\mathrm{NO}_{2}$ através de reação com $\mathrm{O}_{3}$, e durante as horas diurnas o $\mathrm{NO}_{2}$ é convertido de volta para $\mathrm{NO}$ como resultado da fotólise, o que leva à regeneração de $\mathrm{O}_{3}$ (Han et al, 2011). Ainda segundo Han et al (2011), que relataram resultados semelhantes, o $\mathrm{O}_{3}$ em atmosferas urbanas atinge picos durante o dia, geralmente entre as 14:00 e 15: 00, quando ocorre o máximo na intensidade de radiação solar e da temperatura. Esse aumento provavelmente é marcado pela fotólise do $\mathrm{NO}_{2}$ e pela expansão da altura da camada limite durante o dia, que pode resultar na mistura de $\mathrm{O}_{3}$ devido à estratificação térmica e à transferência de calor por convecção para a superfície do ar em altitudes mais elevadas (Swamy et al, 2012). Após atingir a concentração máxima às 14:00, a concentração de $\mathrm{O}_{3}$ diminui lentamente, devido à diminuição da atividade fotoquímica.

As concentrações de $\mathrm{O}_{\mathrm{x}}$ representadas na Figura 2 estão "marcadas" por concentrações de $\mathrm{NO}_{2}$, especialmente no início da manhã, quando as concentrações são majoradas, principalmente devido ao aumento do tráfego de veículos. As maiores concentrações de $\mathrm{O}_{\mathrm{x}}$ ocorreram durante o período da tarde, revelando assim uma influência dos processos fotoquímicos (Han et al, 2011; Notario et al, 2012). À noite, o $\mathrm{O}_{\mathrm{x}}$ diminui devido à ausência de radiação solar. Essa falta de radiação dificulta a formação de $\mathrm{NO}_{2}$ e de $\mathrm{O}_{3}$ por reações fotolíticas, bem como as reações de $\mathrm{NO}_{2}$ com $\mathrm{NO}_{3}$ e de $\mathrm{NO}_{2}$ com $\mathrm{O}_{3}$ (Jenkin e Clemitshaw, 2000). Os dados relatados no presente estudo são consistentes com os de vários estudos (Park et al, 2008; Hagler et al, 2009; Teixeira et al, 2009; Carslaw et al, 2011), que descrevem a influência das emissões veiculares no aumento da concentração de alguns poluentes, principalmente óxido de nitrogênio.

Os níveis de $\mathrm{SO}_{2}$ aumentam ligeiramente nas horas centrais do dia, embora esses níveis estejam muito próximos do limite de detecção, de modo que esses incrementos se encontram na mesma ordem de grandeza de erro de medição. Esses aumentos ao meio-dia também são detectados nas concentrações de $\mathrm{NO}_{\mathrm{x}}$ e podem estar associados à evolução da altura do Camada Limite Planetária (CLP). O desenvolvimento de uma CLP instável com aumento da altitude nas horas centrais facilita o transporte de poluentes de áreas urbanas e industriais para regiões de fundo que se encontram na direção dos ventos predominantes no topo dessa CLP. Em alguns parques, os valores máximos de $\mathrm{NO}_{\mathrm{x}}$ são observados pela manhã e à noite. Esses estão associados às emissões de veículos transitando nas horas de pico, indicando exposição ao tráfego. 
A variabilidade diária de $\mathrm{O}_{3}$ é condicionada por fatores meteorológicos e padrões de emissões de gases precursores. Os picos de concentração são observados durante o período vespertino, após a atividade fotoquímica máxima. As reduções das concentrações durante a noite não são particularmente marcadas, elas respondem a processos envolvendo massas de ar previamente carregadas com $\mathrm{O}_{3}$ produzidas local ou regionalmente durante o dia. Essas massas de ar sofrem recirculação e geram concentrações notáveis de ozônio em áreas remotas durante a noite (Escudero et al, 2014).

Os coeficientes de correlação de Pearson entre os poluentes atmosféricos e os parâmetros meteorológicos medidos durante o ano de 2015 são apresentados na Tabela 4. A análise estatística revelou a existência de uma correlação positiva significativa entre a concentração de ozônio e a radiação total, a radiação UV e a temperatura: $\mathrm{R}=0,618 ; 0,625$ e 0,642 para $\mathrm{p}<0,001$, respectivamente. Existe uma correlação negativa significativa entre a concentração de ozônio e a umidade relativa $(R=-0,703 ; p<0,001)$. As dependências da concentração de ozônio com a radiação solar, com a temperatura e com a umidade relativa, considerando os preditores mais importantes influenciando a variação de seu conteúdo no ar ambiente, estão apresentadas na Tabela 4. Os valores obtidos do coeficiente de determinação $\left(\mathrm{R}^{2}\right)$ para as dependências da concentração de ozônio com a radiação solar, com a temperatura e com a umidade relativa são 0,38; 0,41 e 0,49, respectivamente. Esses valores indicam grandes influências catalíticas de todos os três parâmetros na produção troposférica de ozônio. Esses resultados encontram-se dentro do mesmo intervalo de correlação que os reportados por Gonzales et al, (2010) e Martin et al, (2010).

As concentrações de $\mathrm{SO}_{2}$, de $\mathrm{NO}, \mathrm{NO}_{2}$ e $\mathrm{NOx}$ têm correlação negativa com a concentração de ozônio: $R=-0,032$ e $p<0,01 ; R=-0,312$ e $p<0,01 ; R=-0,103$ e p < 0,01 e $\mathrm{R}=-0,305$ respectivamente, e uma correlação positiva para o $\mathrm{O}_{\mathrm{x}}$ : $\mathrm{R}=0,855$ e $\mathrm{p}<0,001$. Os resultados obtidos estão de acordo com os valores relatados por Lengyel et al, (2004). Por outro lado, suas correlações com $\mathrm{NO}, \mathrm{NO}_{2}$ e $\mathrm{NO}_{\mathrm{x}}$ são altamente positivas: $\mathrm{R}=0,748$ e $\mathrm{p}<$ 0,$01 ; \mathrm{R}=0,844$ e $\mathrm{p}<0,01 ; \mathrm{R}=0,844$ e $\mathrm{p}<0,01$ respectivamente, indicando que todos esses gases são originários da mesma fonte. $O$ único preditor que não tem correlação estatisticamente significativa com a concentração de ozônio é a velocidade do vento. Considerando as condições climáticas, existe uma grande correlação positiva entre a temperatura do ar e a radiação solar, por um lado, e a sua correlação negativa com a umidade relativa. A correlação entre umidade relativa e radiação solar também é negativa. 
A Tabela 4 mostra os coeficientes de correlação entre as concentrações dos poluentes $\mathrm{NO}, \mathrm{NO}_{2}, \mathrm{NO}_{\mathrm{x}}, \mathrm{O}_{3}, \mathrm{O}_{\mathrm{x}}$ e os parâmetros meteorológicos medidos para a cidade de Campo Grande. Foram encontradas correlações positivas $(>0,5)$ entre os óxidos de nitrogênio (NO, $\mathrm{NO}_{2}, \mathrm{NOx}$ ), evidenciando que esses poluentes tinham a mesma fonte e confirmando a influência de fontes móveis. Os óxidos de nitrogênio apresentam correlação significativa com o ozônio $(<0,5)$, embora inversa. Esses resultados são semelhantes aos obtidos por He e Lu (2012). Por ser um poluente fotoquímico, o ozônio também teve uma correlação significativa com a radiação solar e a temperatura. Essas relações de ozônio também foram descritas nos estudos de Teixeira et al (2009) e Pudasainee et al (2006). Além disso, os resultados do presente estudo mostraram correlação do $\mathrm{O}_{\mathrm{x}}$ com a radiação solar e a temperatura ambiente, indicando a influência dos processos fotoquímicos neste poluente em áreas urbanas (Han et $a l, 2011)$.

Tabela 4. Os coeficientes de correlação de Pearson entre os poluentes atmosféricos e os parâmetros meteorológicos medidos durante o ano de 2015.

\begin{tabular}{|c|c|c|c|c|c|c|c|c|c|c|c|c|}
\hline & $\mathrm{O}_{3}$ & $\mathrm{SO}_{2}$ & $\mathrm{NO}$ & $\mathrm{NO}_{2}$ & $\mathrm{NO}_{\mathrm{x}}$ & $\mathrm{O}_{\mathrm{x}}$ & Temp. & Umid. & Press. & $\begin{array}{l}\text { Vel. } \\
\text { Vent. }\end{array}$ & $\begin{array}{l}\text { Dir. } \\
\text { Vent. }\end{array}$ & $\operatorname{RadG}$ \\
\hline $\mathrm{SO}_{2}$ & $-0,032$ & $* *$ & $* *$ & $* *$ & *** & *** & $* *$ & ** & $* *$ & $* *$ & $* *$ & ** \\
\hline $\mathrm{NO}$ & $-0,312$ & 0,099 & $* *$ & *** & *** & *** & $* *$ & $* *$ & $* *$ & $* *$ & $* *$ & $* *$ \\
\hline $\mathrm{NO}_{2}$ & $-0,103$ & 0,156 & 0,174 & ** & $* *$ & *** & $* *$ & $* *$ & $* *$ & $* *$ & $* *$ & ** \\
\hline $\mathrm{NO}_{\mathrm{x}}$ & $-0,305$ & 0,148 & 0,914 & 0,559 & $* *$ & $* *$ & $* *$ & $* *$ & $* *$ & $* *$ & $* *$ & $* *$ \\
\hline $\mathrm{O}_{\mathrm{x}}$ & 0,855 & 0,053 & $-0,192$ & 0,428 & 0,015 & $* *$ & $* *$ & $* *$ & $* *$ & $* *$ & $* *$ & $* *$ \\
\hline Temp. & 0,642 & 0,042 & $-0,15$ & 0,111 & $-0,08$ & 0,641 & $* *$ & $* *$ & $* *$ & $* *$ & $* *$ & $* *$ \\
\hline Umid. & $-0,703$ & $-0,023$ & 0,032 & $-0,005$ & 0,024 & $-0,642$ & $-0,743$ & $* *$ & $* *$ & $* *$ & $* *$ & $* *$ \\
\hline Press. & $-0,082$ & 0,08 & 0,097 & $-0,166$ & 0,013 & $-0,161$ & $-0,358$ & 0,019 & $* *$ & $* *$ & $* *$ & ** \\
\hline $\begin{array}{l}\text { Vel. } \\
\text { Vent. }\end{array}$ & 0,456 & 0,029 & $-0,281$ & $-0,12$ & $-0,286$ & 0,352 & 0,249 & $-0,257$ & $-0,034$ & $* *$ & $* *$ & $* *$ \\
\hline $\begin{array}{l}\text { Dir. } \\
\text { Vent. }\end{array}$ & 0,115 & $-0,045$ & $-0,181$ & $-0,219$ & $-0,242$ & $-0,01$ & $-0,001$ & $-0,049$ & $-0,047$ & 0,07 & $* *$ & $* *$ \\
\hline RadG & 0,618 & 0,035 & $-0,037$ & $-0,05$ & $-0,052$ & 0,536 & 0,637 & $-0,729$ & $-0,01$ & 0,245 & 0,034 & ** \\
\hline
\end{tabular}




\begin{tabular}{|l|l|l|l|l|l|l|l|l|l|l|l|l|}
\hline UV & 0,624 & 0,042 & $-0,044$ & $-0,062$ & $-0,063$ & 0,535 & 0,676 & $-0,723$ & $-0,039$ & 0,282 & 0,046 & 0,965 \\
\hline
\end{tabular}

Fonte:elaboração propria

A velocidade dos ventos mostrou uma correlação significativa com o ozônio e uma correlação inversa com os óxidos de nitrogênio, como indicado na Tabela 4. Essa relação é explicada pelo fato que velocidades dos ventos mais altas aumentam a dispersão e a mistura desses poluentes atmosféricos emitidos em fontes mais próximas, isto é, em rodovias e fontes estacionárias, otimizando assim a formação do ozônio a partir de precursores. Ventos muito fortes, no entanto, fogem à essa regra. Estes dados mostram concordância com outros estudos, como os apresentados por Jones et al (2010) e Guerra e Miranda (2011).

As correlações de óxidos de nitrogênio $\left(\mathrm{NO}, \mathrm{NO}_{2}, \mathrm{NO}_{\mathrm{x}}\right)$ com a umidade relativa foram significativas ao nível de 0,01 . Isso pode ser atribuído à influência da migração de novas massas de ar sobre a área de estudo, principalmente as massas de ar polares, que podem trazer ar limpo atmosférico e minimizar as concentrações cumulativas (de fundo) desses poluentes. Esses resultados são semelhantes aos relatados em outros estudos, como os de Elminir (2005) e Mavroidis e Ilia (2012).

Além disso, a Tabela 4 mostra a correlação negativa obtida entre o $\mathrm{O}_{3}$ com a umidade relativa do ar. Essa relação pode ser explicada pelo fato de que quando a umidade relativa aumenta, os principais caminhos fotoquímicos para a remoção de $\mathrm{O}_{3}$ são reforçados (Reddy et $a l$, 2012). No entanto, altos valores de umidade relativa podem estar associados à instabilidade atmosférica e grande cobertura de nuvens, o que pode retardar processos fotoquímicos, com o $\mathrm{O}_{3}$, que acaba sendo esgotado por deposição úmida (Nishanth et al, 2012), e também pelo fato de que ambos têm um comportamento oposto. A umidade relativa tem seu pico noturno com a diminuição da temperatura do ar, enquanto o ozônio mostra seu valor mínimo devido à ausência de radiação solar, e vice-versa. Agudelo-Castaneda et al (2013) também relataram esse comportamento. Song et al (2011) relataram em seu trabalho resultados semelhantes, alta umidade relativa pode retardar a produção de $\mathrm{O}_{3}$.

Os óxidos de nitrogênio, principalmente $\mathrm{NO}_{2}$, apresentaram uma correlação negativa e positiva $(<0,5)$ com a temperatura, respectivamente. Essas relações podem ser explicadas pelo fato de que os $\mathrm{NO}_{x}$ favorecem a produção de $\mathrm{O}_{3}$ na presença de luz solar e altas temperaturas (Jacib e Winner, 2009). 


\section{Relação entre $\mathrm{NO}_{2}, \mathrm{NO}_{x}$ e $\mathrm{O}_{x}$}

A análise dos valores da relação $\left[\mathrm{NO}_{2}\right] /\left[\mathrm{O}_{\mathrm{x}}\right]$ foi utilizada para explorar as variações de concentração de $\mathrm{O}_{3}$ ao nível do solo e a relação entre os dados $\mathrm{O}_{x}$ e $\mathrm{NOx}$ (Clapp e Jenkin, 2001; Han et al, 2011). Existe uma relação de estado fotoestacionária entre $\mathrm{NO}, \mathrm{NO}_{2}$ e $\mathrm{O}_{3}$ (Clapp e Jenkin, 2001; Han et al, 2011). Com base nisso, pode-se inferir uma variação esperada. A Figura 3 mostra a análise de regressão com média diária da relação $\left[\mathrm{NO}_{2}\right] /\left[\mathrm{O}_{\mathrm{x}}\right]$ como função de $\mathrm{NO}_{\mathrm{X}}$. Os dados revelam que, para valores mais baixos da relação $\left[\mathrm{NO}_{2}\right] /\left[\mathrm{O}_{\mathrm{x}}\right]$, existem valores baixos de $\mathrm{NO}_{\mathrm{x}}$, o que implica que, nesses instantes, as concentrações de $\mathrm{O}_{\mathrm{x}}$ são predominantemente marcadas por altas concentrações de $\mathrm{O}_{3}$. Além disso, com o aumento das concentrações de $\mathrm{NO}_{\mathrm{x}}$, uma grande parte do $\mathrm{O}_{\mathrm{x}}$ está presente na forma de $\mathrm{NO}_{2}$. Os valores elevados de $\left[\mathrm{NO}_{2}\right] /\left[\mathrm{O}_{\mathrm{x}}\right]$ também podem ser explicados pelo processo de oxidação de $\mathrm{NO}$ a $\mathrm{NO}_{2}$, com concentrações de $\mathrm{NO}_{\mathrm{x}}$ marcadas principalmente pela concentração de $\mathrm{NO}_{2}$.

As equações apresentam valor de intercepção de $-0,46 ; \mathrm{R}^{2}=55.7 \%$ e $\mathrm{p}<0.00001$. No entanto, estudos adicionais devem ser realizados para entender de forma mais precisa e determinada a relação entre $\mathrm{NO}, \mathrm{NO}_{2}$ e $\mathrm{O}_{3}$, a fim de se obter funções adequadas.

A exposição da população a níveis elevados de poluentes por vários meses carece de uma investigação mais aprofundada, utilizando uma análise de risco adequada ao ser humano. Além disso, o $\mathrm{O}_{3}$ tem o potencial de ser um problema no futuro com o aumento das atividades antropogênicas (Souza et al, 2016).

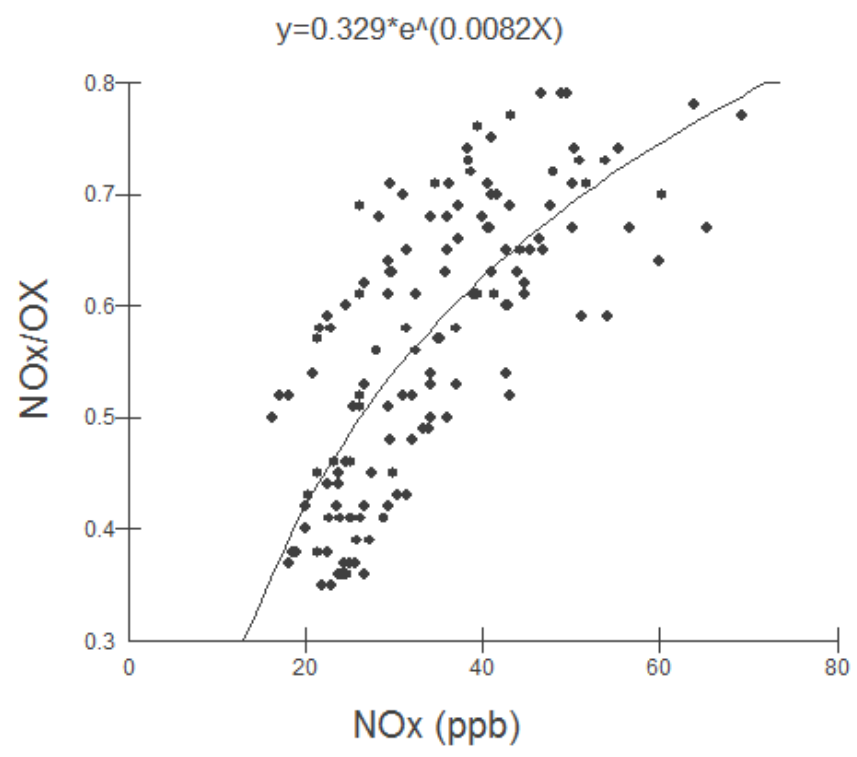

Figura 3 - Médias diárias da razão de concentração de $\mathrm{NO}_{\mathrm{x}} / \mathrm{O}_{\mathrm{x}}$ e de $\mathrm{NO}_{\mathrm{x}}$ 


\section{CONCLUSÃO}

A componente sazonal mostrou concentrações de $\mathrm{NO}, \mathrm{NO}_{2}$ e $\mathrm{NO}$ x acima da média durante o inverno, devido a condições meteorológicas adversas, enquanto as concentrações de $\mathrm{O}_{3}$ foram acima da média durante o verão, devido ao aumento da atividade fotoquímica. As correlações positivas $(>0,5)$ entre óxidos de nitrogênio $\left(\mathrm{NO}, \mathrm{NO}_{2}, \mathrm{NO}_{\mathrm{X}}\right)$ indicam que esses poluentes provêm da mesma fonte, evidenciando a influência de fontes móveis em Campo Grande. $\mathrm{Na}$ correlação de $\left[\mathrm{NO}_{2}\right] /\left[\mathrm{O}_{\mathrm{x}}\right]$ com $\mathrm{NO}_{\mathrm{x}}$, observou-se que, para baixos valores de $\left[\mathrm{NO}_{2}\right] /\left[\mathrm{O}_{\mathrm{x}}\right]$, existem baixos valores de $\mathrm{NO}_{\mathrm{x}}$, indicando que as concentrações são predominantemente marcadas por $\mathrm{O}_{3}$. Para valores elevados de $\left[\mathrm{NO}_{2}\right] /\left[\mathrm{O}_{\mathrm{x}}\right]$, as concentrações de $\mathrm{NO}_{\mathrm{x}}$ foram marcadas principalmente por concentrações de $\mathrm{NO}_{2}$, o que pode ser explicado pelo processo de oxidação de $\mathrm{NO}$ e $\mathrm{NO}_{2}$.

$\mathrm{O}$ presente estudo analisou as concentrações de $\mathrm{NO}, \mathrm{NO}_{2}, \mathrm{NOx}$ e $\mathrm{O}_{3}$ medidas na cidade de Campo Grande durante um ciclo completo de 365 dias (medidas horárias e diárias), para o ano específico de 2015. Os resultados indicam que o ciclo diurno da concentração de ozônio tem um pico ao meio dia. A concentração de ozônio aumenta lentamente após o nascer do sol, atingindo um máximo durante o dia e depois diminui até a manhã seguinte. Isso é devido à formação fotoquímica do $\mathrm{O}_{3}$. A forma e a amplitude dos ciclos de ozônio são fortemente influenciadas pelas condições meteorológicas e pelos níveis prevalentes de precursores $\left(\mathrm{NO}_{\mathrm{x}}\right)$. Na região de estudo, o ciclo diário de concentração de NO decorre de emissões de veículos, e sua conversão para $\mathrm{NO}_{2}$, tendo um grande impacto no ciclo diário de níveis de ozônio. É interessante, em estudos futuros, analisar a relação entre $\mathrm{NO}_{2}$ e $\mathrm{NO}_{\mathrm{x}}$, bem como $\mathrm{NO}$ e $\mathrm{NO}_{x}$, e entre $\mathrm{O}_{3}$ e $\left[\mathrm{NO}_{2}\right] /[\mathrm{NO}]$, o que poderia ser útil na previsão de $\mathrm{O}_{3}$ e estratégias de controle de poluição do ar. Além disso, as relações entre UV e $\mathrm{O}_{3}$ durante o dia ainda não são bem compreendidas e justificam um estudo mais aprofundado.

O padrão diurno da concentração de ozônio superficial indica claramente sua dependência do processo fotoquímico, processo de produção, enquanto as variações sazonais foram principalmente devidas à fotoquímica. A evolução diurna dos $\mathrm{NO}_{\mathrm{x}}$ também apresenta diferenças sazonais. Conforme mencionado anteriormente, os níveis obtidos durante o inverno são maiores do que os obtidos durante o verão. 


\section{REFERÊNCIAS BIBLIOGRÁFICAS}

Agudelo-Castaneda, D. M.; Teixeira, E. C.; Rolim, S. B. A.; Pereira, F. N.; Wiegand, F. Measurement of particle number and related pollutant concentrations in an urban area in South Brazil. Atmospheric environment, V. 70, p. 254-262, 2013.

Brasil; Ministério do Meio Ambiente. Resolução CONAMA 3/1990. Brasília, 1990.

Carslaw D. C.; Beevers S. D.; Tate J. E.; Westmoreland E. J.; Williams M. L. Recent evidence concerning higher $\mathrm{NO}_{\mathrm{x}}$ emissions from passenger cars and light duty vehicles. Atmospheric environment, V. 45, N. 39, p. 7053-7063, 2011.

Carslaw, D. C. Evidence of an increasing $\mathrm{NO}_{2} / \mathrm{NO}_{\mathrm{x}}$ emissions ratio from road traffic emissions. Atmospheric environment, V. 39, N. 26, p. 4793-4802, 2005.

Clapp, L. J.; Jenkin, M. E. Analysis of the relationship between ambient levels of $\mathrm{O}_{3}$, $\mathrm{NO}_{2}$ and $\mathrm{NO}$ as a function of $\mathrm{NO}_{\mathrm{x}}$ in the UK. Atmospheric environment, V. 35, p. 6391-6405, 2001.

Coronado C. R.; Carvalho J. A.; Silveira J. L. Biodiesel $\mathrm{CO}_{2}$ emissions: A comparison with the main fuels in the Brazilian Market. Fuel Processing Technology, V. 90, N. 2, p. 204-211, 2009.

Dallarosa, J. B.; Teixeira, E. C.; Alves, R. C. M. Application of numerical models in the formation of ozone and its precursors in areas of influence of coal-fired power station Brazil. Water air and soil pollution, V. 178, N. 1-4, p. 385-399, 2007.

E. C. Directive 2008/50/EC of the European Parliament and of the Council on ambient air quality and cleaner air for Europe. Official journal, V. 152, p. 1-44, 2008.

Elminr, H. K. Dependence of urban air pollutants on meteorology. Science of the total environment, V. 350, N. 1-3, p. 225-237, 2005.

Escudero, M.; Lozano, A.; Hierro, J.; Del Valle, J.; Mantilla, E. Urban influence on increasing ozone concentrations in a characteristic Mediterranean agglomeration. Atmospheric environment, V. 99, p. 322-332, 2014.

Geddes, J. A.; Murphy, J. G.; Wang, D. K. Long term changes in nitrogen oxides and volatile organic compounds in Toronto and the challenges facing local ozone control. Atmospheric environment, V. 43, N. 21, p. 3407-3415, 2009.

Gonzales, L.; Bermejo, R. O.; Parra, A.; Elustondo, D.; Garrigo, J.; Santamaria. J. M. Rural $\mathrm{O}_{3}$ levels in the Middle Ebro Basin during the plant growing season. Water air soil pollut. V. 226, N. 1, p. 23-34, 2010.

Guerra, F. P.; Miranda, R. M. Influence of meteorology in the concentration of atmospheric pollutant $\mathrm{PM}_{2.5}$ in RJRM and MRSP. Proceedings of the II Congress Brazilian Environmental Management, Parana, Brazil, p. 6-9, 2011.

Hagenbjörk, A.; Malmqvist, E. K.; Sommar, N. J.; Modig, L. The spatial variation of $\mathrm{O}_{3}$, NO, $\mathrm{NO}_{2}$ and $\mathrm{NO}_{x}$ and the relation between them in two Swedish cities. Environ monit. assess., V. 189, N. 4, p. 161, 2017.

Hagler, G. S. W.; Baldauf, R. W.; Thoma, E. D.; Long, T. R.; Snow, R. F.; Kinsey, J. S.; Oudejans, L.; Gullett, B. K. Ultrafine particles near a major roadway in Raleigh, North Carolina: downwind attenuation and correlation with traffic-related pollutants. Atmospheric environment, V. 43, N. 6, p. 1229-1234, 2009.

Han, S. Q.; Bian, H.; Feng, Y. C.; Liu, A. X.; Li, X. J.; Zeng, F.; Zhang, X. L. Analysis of the relationship between $\mathrm{O}_{3}, \mathrm{NO}$ and $\mathrm{NO}_{2}$ in Tianjin, China. Aerosol and air quality research, $\mathrm{V}$. 11, p. 128-139, 2011.

He, H. D.; Lu, W. Z. Decomposition of pollution contributors to urban ozone levels concerning regional and local scales. Building and environment, V. 49, p. 97-103, 2012. 
Jacob, D. J.; Winner, D. A. Effect of climate change on air quality. Atmospheric environment, V. 43, N. 1, p. 51-63, 2009.

Jenkin, M. E.; Clemitshaw, K. C. Ozone and other secondary photochemical pollutants: chemical processes governing their formation in the planetary boundary layer. Atmospheric environment, V. 34, N. 16, p. 2499-2527, 2000.

Jones, A. M.; Harrison, R. M.; Baker, J. The wind speed dependence of the concentrations of airborne particulate matter and NOx. Atmospheric environment, V. 44, N. 13, p. 1682-1690, 2010.

Lenguyel, A.; Heberger, K.; Paksy, L.; Banhidi, O.; Rajko, R. Prediction of ozone concentration in ambient air using multivariate methods. Chemosphere, V. 57, N. 8, p. 889896, 2004.

Martin, P.; Cabanas, B.; Villanueva, F.; Gallero, P. M.; Colmenar, I.; Salgado, S. Ozone and nitrogen dioxide levels monitored in an urban area (ciudad real) in central-southern Spain. Water, air and soil pollution, V. 208, p. 305-316, 2010.

Mavroidis, I.; Ilia, M. trends of $\mathrm{NO}_{x}, \mathrm{NO}_{2}$ and $\mathrm{O}_{3}$ concentrations at three different types of air quality monitoring stations in Athens, Greece. Atmospheric environment, V. 63, p. 135-147, 2012.

Monteiro, A.; Vautard, R.; Borrego, C.; Miranda A. I. Long-term simulations of photo oxidant pollution over Portugal using the CHIMERE model. Atmospheric environment, V. 39, N. 17, p. 3089-3101, 2005.

Moura, M. A. L.; Meixner, F. X.; Trebs, I.; Molion, L. C. B.; Nascimento Filho, M. F. Medições de $\mathrm{NO}-\mathrm{NO}_{2}-\mathrm{O}_{3}$ na Amazônia central durante o experimento LBA / Claire 2001.

Revista brasileira de meteorologia, V. 19, N. 1, p. 49-58, 2004.

Nishanth, T.; Kumar, M. K. S.; Valsaraj, K. T. Variations in surface ozone and $\mathrm{NO}_{\mathrm{x}}$ at Kannur: a tropical, coastal site in India. Journal of atmospheric chemistry, V. 69, N. 2, p. 101-126, 2012.

Notario, A.; Bravo, I.; Adame, J. A.; Diaz-de-Mera, Y.; Aranda, A.; Rodriguez, A.; Rodriguez, D. Analysis of $\mathrm{NO}, \mathrm{NO}_{2}, \mathrm{NO}_{\mathrm{x}}, \mathrm{O}_{3}$ and oxidant $\left(\mathrm{O}_{\mathrm{x}}=\mathrm{O}_{3}+\mathrm{NO}_{2}\right)$ levels measured in a metropolitan area in the southwest of Iberian Peninsula. Atmospheric research, V. 104-105, p. 217-226. 2012.

Papanastasiou, D. K.; Melas, D.; Bartzanas, T.; Kittas C. Estimation of ozone trend in central Greece, based on meteorologically adjusted time series. Environmental modeling \& assessment, V. 17, N. 4, p. 353-361, 2012.

Park, K.; Park, J. Y.; Kwak, J. H.; Cho, G. N.; Kim, J. S. Seasonal and diurnal variations of ultrafine particle concentration in urban Gwangju, Korea: observation of ultrafine particle events. Atmospheric environment, N. 42, p. 788-799, 2008.

Pires, J. C. M.; Souza, A.; Pavão, H. G.; Martins, F. G. Variation of surface ozone in Campo Grande, Brazil: meteorological effect analysis and prediction. Environmental science and pollution research international, V. 21, n. 17, p. 10550-10559, 2014.

Pudasainee, D.; Sapkota, B.; Shrestha, M. L.; Kaga, A.; Kondo, A.; Inoue, Y. Ground level ozone concentrations and its association with $\mathrm{NO}_{\mathrm{x}}$ and meteorological parameters in Kathmandu valley, Nepal. Atmospheric environment, V. 40, N. 40, p. 8081-8087, 2006.

Reddy, B. S. K.; Kumar, K. R.; Balakrishnaiah, G.; Gopal, K. R.; Reddy, R. R.; Sivakumar, V.; Lingaswamy, A. P.; Arafath, S. M.; Umadevi, K.; Kumari, S. P.; Ahammed, Y. N.; Lal, S. Analysis of diurnal and seasonal behavior of surface ozone and its precursors $\left(\mathrm{NO}_{\mathrm{x}}\right)$ at a semiarid rural site in southern India. Aerosol and air quality research, V. 12, p. 1081-1094, 2012. 
Sadanaga, Y.; Shibata, S.; Hamana, M.; Takenaka, N.; Bandow, H. Weekday/weekend difference of ozone and its precursors in urban areas of Japan, focusing on nitrogen oxides and hydrocarbons. Atmospheric environment, V. 42, N. 19, p. 4708-4723, 2008.

Sebald, L.; Treffeisen, R.; Reimer, E.; Hies, T. Spectral analysis of air pollutants. Part 2: ozone time series. Atmospheric environment, V. 34, p. 3503-3509, 2000.

Seinfeld, J. H.; Pandis S. N. Atmospheric chemistry and physics: from air pollution to climate change $\left(2^{\text {nd }}\right)$, John Wiley \& sons, New Jersey, US, p.1203, 2006.

Singla, V.; Satsangi, A.; Pachauri, T.; Lakhani, A.; Kumari, K. M. Ozone formation and destruction at a sub-urban site in north central region of India. Atmospheric research, V. 101, p. 373-385, 2011.

Song, F.; Shin, J. Y.; Jusino-Atresino, R.; Gao, Y. Relationships among the springtime ground-level $\mathrm{NO}_{x}, \mathrm{O}_{3}$ and $\mathrm{NO}_{3}$ in the vicinity of highways in the US east coast. Atmospheric pollution research, V. 2, p. 374-383, 2011.

Souza, A.; Aristone, F.; Gonçalves, F. V. Modeling of surface and weather effects ozone concentration using neural networks in west center of Brazil. Journal of climatology \& weather forecasting, V. 3, p. 1-44, 2015.

Souza, A.; Aristone, F.; Kumar, U.; Andric, E. K.; Arsic, M.; Ikefuti, P.; Sabbah, I. Analysis of the correlations between $\mathrm{NO}, \mathrm{NO}_{2}$ and $\mathrm{O}_{3}$ concentrations in Campo Grande, MS, Brazil. Eur. chem. bull., V. 6, p. 284-291, 2017.

Souza, A.; Aristones, F.; Pavão, H. G.; Fernandes, W. A. Development of a short-term ozone prediction tool in Campo Grande - MS, Brazil area based on meteorological variables. Open journal of air pollution, V. 3, N. 2, p. 42-51, 2014.

Souza, A.; Fernandes, W. A. Surface ozone measurements and meteorological influences in the urban atmosphere of Campo Grande. Acta scientarium technology, V. 36, N. 1, p. 141146, 2014.

Souza, A.; Kovac-Matasovi, E.; Matasovic, B.; Markovi, B. Assessment of ozone variations and meteorological influences in west center of Brazil, from 2004 to 2010. Water, air and soil pollution, p. 227-313, 2016.

Souza, A.; Olaofe, Z. O.; Kumar, S. P.; Ikefuti, P.; Nobrega, L.; Sabbah, I. Probability distributions assessment for modeling gas concentration in Campo Grande, MS, Brazil. Eur. chem. bull., V. 6, N. 12, p. 569-578, 2018.

Souza, A.; Pavão, H.; Lastoria, G.; Gabas, S. G.; Cavazzana, G. H. Modelo de Thom para o zoneamento bioclimático de Mato Grosso do Sul. Revista de geografia norte grande (impresa), V. 46, p. 137-147, 2010.

Souza, A.; Santos, D. A. S. Temperature as a risk factor for hospital admissions in 70 cities of MS. Ciência e natura, V. 38, p. 1512-1522, 2016.

Stedman D. H. Photochemical ozone formation, simplified. Environmental chemistry, V. 1, p. 65-66, 2004.

Swamy, Y. V.; Venkanna, R.; Nikhil, G. N.; Chitanya, D. N. S. K.; Sinha, P. R.; Ramakrishna, M.; Rao, A. G. Impact of nitrogen oxides, volatile organic compounds and black carbon on atmospheric ozone levels at a semiarid urban site in Hyderabad. Aerosol and air quality research, V. 12, p. 662-671, 2012.

Teixeira E. C.; Mattiuzi, C. D. P.; Feltes, S.; Wiegand, F.; Santana, E. R. R. Estimated atmospheric emissions from biodiesel and characterization of pollutants in the metropolitan area of Porto Alegre-RS. Anais da academia brasileira de ciências, V. 84, p. 245-261, 2012.

Teixeira, E. C.; Santana, E. R.; Wiegand, F.; Fachel, J. Measurement of surface ozone and its precursors in an urban area in south Brazil. Atmospheric environment, V. 43, p. 2213-2220, 2009. 
Vieira, L. C.; Korf, E. P.; Brandli, L. L. Passive samplers for air quality monitoring in a Brazilian university. Int. j. environment and pollution, V. 53, N. 1/2, p. 148-158, 2013.

Recebido em 25 de julho de 2017.

Aceito em 04 de maio de 2018. 